\title{
Classroom-Based Parent Interview in Psychiatric Mental Health Nursing
}

\author{
Minjeong Kim¹, Michelle Grace Lee ${ }^{2}$ \\ ${ }^{1}$ School of Nursing, San Diego State University, San Diego, USA \\ ${ }^{2}$ Graduate of the School of Nursing, San Diego State University, San Diego, USA \\ Email: minjeong.kim@sdsu.edu
}

How to cite this paper: Kim, M. and Lee, M.G. (2020) Classroom-Based Parent Interview in Psychiatric Mental Health Nursing. Open Journal of Nursing, 10, 613-616. https://doi.org/10.4236/ojn.2020.106042

Received: January 14, 2020

Accepted: June 5, 2020

Published: June 8, 2020

Copyright ( 2020 by author(s) and Scientific Research Publishing Inc. This work is licensed under the Creative Commons Attribution International License (CC BY 4.0).

http://creativecommons.org/licenses/by/4.0/ (c) (i) Open Access

\begin{abstract}
A classroom-based parent interview was designed and implemented in an undergraduate psychiatric mental health nursing class to fill the gap between nursing students and parents of child or adolescent patients with mental health issues faced during clinical. The goals of this learning activity were to increase understanding of what parents experience when dealing with their child's mental health problems and to increase student engagement and attention. The class using this learning activity consisted of three parts: 1) an assigned pre-class reading; 2) a mini-lecture; and 3) a parent interview presentation. Students were pre-assigned a reading chapter and faculty-developed interview questions. During the mini-lecture, important knowledge related to mental health care of children was assessed using CourseKey software. After the mini-lecture, the parent guest speaker delivered her presentation about herself, her child's strengths and abilities, her child's mental health problems and their impact, and her family's strengths based on the interview question prompts for about 30 minutes, followed by 20 minutes of a Q \& A session. The set of interview questions was developed by faculty based on the competency questions of the Child Behavior Check List. Despite some limitations, this classroom-based parent interview using a flipped classroom model was found to be a meaningful learning strategy by increasing student engagement and attention, increasing retention of knowledge learned in class, and filling that gap in clinical.
\end{abstract}

\section{Keywords}

Nursing Education, Parent Interview, Psychiatric Mental Health Nursing

\section{Introduction}

One of the challenges for the undergraduate psychiatric mental health nursing 
clinical is the lack of interaction between nursing students and the parents or family members of child and adolescent patients with mental health problems. This can be explained by several factors such as a limited access to parents due to the timing of clinical hours and changes in hospital atmosphere, a lack of professional relationship building skills, a lack of parent interviewing skills, and student anxiety. Therefore, in order to fill that gap in clinical, a classroom-based parent interview presentation was designed and implemented under the topic, "Caring for Children with Mental Illness," as one of the learning activities in class. The goals of this activity were to increase understanding of what families experience when dealing with their children's mental health problems and to increase student engagement and attention.

\section{Methods}

This learning activity included about 80 undergraduate nursing students who took their psychiatric mental health nursing lecture and clinical in the Fall 2017 semester. The two and a half hours-long class utilizing this activity consisted of three parts: 1) an assigned pre-class reading, 2) a mini-lecture, and 3) a parent interview presentation. In detail, one week prior to the scheduled lecture and parent interview presentation, students were asked to read an assigned chapter in the textbook along with the faculty-developed interview questions. During the mini-lecture, questions were asked through CourseKey [1] to check whether the students understood important concepts related to children's mental health care from the assigned reading. The CourseKey software delivers in-class, auto-graded quizzes for students to complete and review their scores. Each question was answered by students and immediate feedback was given by faculty based on the poll results provided by CourseKey. Lastly, the parent guest speaker delivered her presentation about herself, her child's strengths and abilities, her child's mental health problems and their impact, and her family's strengths based on the faculty-developed PowerPoint interview question prompts for about 30 minutes, followed by 20 minutes of a $\mathrm{Q} \& \mathrm{~A}$ session.

In order to maximize student learning from the parent guest speaker's presentation in this large size class, the speaker was selected based on her background of successfully dealing with her child's mental health problems and having a child who had been hospitalized in a psychiatric inpatient unit where our nursing students currently have clinical and has been recovered. In addition, a structured interview style presentation was preferred rather than one-on-one parent-student interviews. A set of interview questions was formulated by faculty based on the competency questions of the Child Behavior Check List (CBCL) [2], made into a PowerPoint, and provided to the speaker in advance so that she could prepare for her presentation in class. Interview questions included: 1) About You: What's your age, gender, race/ethnicity, and religion? What's your job or talent? What's your education \& socioeconomic level? How can you describe your physical and mental health conditions?; 2) About Your Child: What's his age, gender, ethnicity, and religion? How can you describe your son's perso- 
nality? What kind of sports does your son most like to take part in? What's your son's favorite hobbies, activities, and games, other than sports? What organizations, clubs, teams, or groups does your son belongs to? How well does your son get along with his brothers, sisters, and parents? How well does your son get along with his friends? How many close friends does your son have? How many times a week does your son do things with friends outside of regular school hours? How can you describe your son's performance in academic subjects?; 3) About Your Child's Mental Health Problems and Their Impact: Could you describe your son's mental health problems? How has your son's mental health condition affected you in your own life or the lives of other family members (financial/housing situation, work or social life, relationships with family and friends or neighbors or other members of your community, and your own physical and mental health conditions)?; and 4) Your Family's Strength: What are some of your family's strengths in dealing with your son's mental health problems? In conclusion, how might nurses or other health care professionals help other family members like you?

\section{Results and Discussion}

In this learning activity, the students were very interested in meeting the speaker and learning from her personal experiences. This could especially be seen during the Q \& A session when the students eagerly asked many insightful questions. Students stated that the speaker allowed them to better understand and empathize with what patients and their families are going through, thus enabling better patient care. They also mentioned that this session was deeply helpful and deserved to continue on in the next semester. Therefore, this activity was found to be a meaningful learning strategy by increasing student engagement and attention, increasing retention of knowledge learned in class, and filling that gap in clinical. However, this classroom-based parent interview using a flipped classroom model did not provide opportunities for in-depth and one-on-one interactions between the speaker and students despite active participation in the Q \& A session. Only one speaker was recruited in this learning activity which allowed students to focus on her stories, but it would be more beneficial if a group of parents with children and adolescents who have diverse backgrounds and mental health issues could be invited to provide a richer learning environment for the students. If possible, recruiting both parents and their children or adolescents would be even better for students to observe parent-child interactions that are rarely seen during the psychiatric mental health nursing clinical.

\section{Acknowledgements}

The authors would like to acknowledge undergraduate students in the School of Nursing, San Diego State University and the guest speaker for their participation in this innovative learning activity which is a part of the psychiatric mental health nursing class. 


\section{Conflicts of Interest}

The authors declare no conflicts of interest regarding the publication of this paper.

\section{References}

[1] CourseKey. https://coursekey.com/

[2] Achenbach, T.M. and Rescorla, L.A. (2001) Manual for the ASEBA School-Age Forms \& Profiles. University of Vermont Research Center for Children, Youth, \& Families, Burlington. 